\title{
Determinación de los parámetros de la curva de brillo de nanocristales de óxidos de itrio por el método de los tres puntos
}

\author{
Francisco del Rosario Sánchez ${ }^{1}$, Modesto Sosa ${ }^{2 *}$ \\ ${ }^{1}$ Universidad APEC, Escuela de Ingeniería, Santo Domingo, \\ República Dominicana \\ ${ }^{2}$ Universidad de Guanajuato, Campus León, \\ División de Ciencias e Ingenierías, León, Gto., México \\ modesto@fisica.ugto.mx
}

\begin{abstract}
Resumen. Se ha estudiado la respuesta termoluminiscente de muestras de óxido de itrio $\left(\mathrm{Y}_{2} \mathrm{O}_{3}\right)$ irradiadas con rayos $\mathrm{X}$, aplicando un rango de exposición entre 17.7 y $40 \mathrm{R}$ y se han determinado los parámetros que caracterizan el orden de la cinética del material y la profundidad de trampas. Se empleó el método de los tres puntos para analizar la curva de brillo, la cual se reconstruyó a partir de un proceso de deconvolución desarrollado en este trabajo usando el software WebPlotDigitizer v3.8. Se empleó el método de los tres puntos para obtener los parámetros cinéticos. Se determinó que los materiales estudiados presentan una cinética de primer orden. La energía de activación se encontró con valores cercanos a $1 \mathrm{eV}$, lo cual está en concordancia con los reportados en la literatura para la mayoría de los materiales termoluminiscentes.
\end{abstract}

Palabras clave: óxido de itrio, curva de brillo, parámetros cinéticos.

\section{Determination of the Glow Curve Parameters of Itrium Oxide Nanocrystals by the Three Point Method}

\begin{abstract}
The thermoluminescent response of samples of yttrium oxide (Y2O3) irradiated with X-rays has been studied, applying a range of exposure between 17.7 and $40 \mathrm{R}$ and the parameters that characterize the order of the kinetics of the material and the depth of traps have been determined. The three point method was used to analyze the glow curve, which was reconstructed from a deconvolution process developed in this work using the WebPlotDigitizer v3.8 software. The three-point method was used to obtain the kinetic parameters. It was determined that the studied materials show a kinetics of first order. The activation energy was found with values close to $1 \mathrm{eV}$, which is in agreement with those reported in the literature for most thermoluminescent materials.
\end{abstract}

*Autor para correspondencia: M. Sosa. 
Keywords: yttrium oxide, glow curve, kinetic parameters.

\section{Introducción}

La termoluminiscencia (TL) es la emisión de luz por ciertos materiales al ser calentados por debajo de su temperatura de incandescencia, habiendo sido previamente expuestos a un agente excitante tal como las radiaciones ionizantes. La luz emitida tiene una longitud de onda mayor que la radiación incidente (ley de Stoke); además, la longitud de onda de la luz emitida es una característica del material luminiscente.

La principal aplicación práctica de la TL es sin duda en la dosimetría de radiaciones o dosimetría termoluminiscente (TLD, por sus siglas en inglés). En esta actividad la TL ha alcanzado un gran desarrollo y un elevado grado de aceptación entre la comunidad científica internacional desde sus inicios [1].

Los materiales termoluminiscentes son ampliamente utilizados en las aplicaciones de Física Médica para determinar la dosis absorbida, tanto en pacientes como en el personal ocupacionalmente expuesto (POE). Asimismo, son de uso común en mediciones de los niveles de radiactividad ambiental en instalaciones hospitalarias. Las medidas de las dosis in vivo proporcionan una manera fiable y eficaz de verificar la exactitud global del proceso en los tratamientos de radiación [2], donde la dosis absorbida no se puede desviar más de un $5.0 \%$ del valor prescrito, según las recomendaciones de la Agencia Internacional de Energía Atómica. La dosimetría in vivo proporciona realmente la dosis absorbida por los pacientes en las sesiones de tratamiento, lo que ayuda a descubrir y limitar los errores en los procedimientos terapéuticos [3]. Las muestras termoluminiscentes son muy convenientes para este uso, particularmente en los procedimientos de radioterapia [4,5].

Los modelos para el estudio de las propiedades dosimétricas de los diferentes materiales TL, se han basado históricamente en la correlación que se observa entre la evolución de los picos TL con la dosis recibida y tasas de calentamiento, entre otros factores, o en las bandas de absorción óptica generadas por la radiación [6]. Se han desarrollado teorías para describir este fenómeno, como son la de cinética de orden uno, dos, general y los diagramas de configuraciones; las cuales han sido tratadas por diferentes autores $[7,8]$. Pero estas teorías resultan muchas veces insuficientes cuando se desea realizar un análisis de la estructura de las curvas termoluminiscentes experimentales (curvas de brillo), por el hecho de que están constituidas por más de una señal TL, lo que hace necesario la implementación de métodos de deconvolución para obtener dichas señales [9-11].

La ventaja del método de deconvolución radica en que proporciona con gran precisión las señales independientes que se encuentran en las curvas de brillo TL experimentales; para lo cual se han desarrollado programas computacionales con ese propósito [12]. Los análisis realizados a curvas TL experimentales por medio de esta técnica y con base en la teoría cinética de orden general muestran cómo las variaciones en un material influyen en la cinética, las profundidades de las trampas y la energía de activación, entre otros parámetros fundamentales, lo cual se refleja en la forma de la curva de brillo [13].

Por su parte, diversos autores [14,15] han reportado las propiedades TL del óxido de itrio $\mathrm{Y}_{2} \mathrm{O}_{3}$, utilizando diversos dopantes y campos de radiación. Se ha demostrado que 
este material presenta una buena respuesta TL a las radiaciones ionizantes, resultando por tanto un buen candidato para dosimetría de radiaciones.

El $\mathrm{Y}_{2} \mathrm{O}_{3}$ es el compuesto del itrio más conocido, probablemente debido a su uso extendido en la industria para la fabricación de sustancias luminiscentes, así como a su elevada estabilidad térmica y una buena transmisión en la gama infraroja en una longitud de onda de 1 a $8 \mu \mathrm{m}$. En estado dopado con tierras raras, como el $\mathrm{Y}_{2} \mathrm{O}_{3}: \mathrm{Eu}^{3+}$, su espectro de excitación presenta una banda en $256 \mathrm{~nm}$ atribuida a una transición por transferencia de carga, así como otros picos angostos y pequeños localizados en 365 , 384 y $395 \mathrm{~nm}$ respectivamente, que corresponden a las transiciones ${ }^{7} \mathrm{~F}_{0} \rightarrow{ }^{5} \mathrm{D}_{4},{ }^{7} \mathrm{~F}_{0} \rightarrow$ ${ }^{5} \mathrm{G}_{3} \mathrm{y}^{7} \mathrm{~F}_{0} \rightarrow{ }^{5} \mathrm{~L}_{6}$ del ion $\mathrm{Eu}^{3+}$, lo cual lo hace un material candidato para dosimetría.

El objetivo de este trabajo fue caracterizar la respuesta termoluminiscente de muestras a base de $\mathrm{Y}_{2} \mathrm{O}_{3}$ irradiadas con rayos $\mathrm{X}$. Se estudiaron muestras de $\mathrm{Y}_{2} \mathrm{O}_{3}$ : $\mathrm{Ce}$, Eu al exponerlas a un campo de radiación de rayos $\mathrm{X}$.

\section{Fundamentos teóricos}

\subsection{Modelo matemático del proceso de TL}

La producción de solamente un tipo de trampas de carga dentro de un material termoluminiscente puede ser descrita por la siguiente expresión [16]:

$$
\frac{d n}{d t}=\Phi f\left(N_{0}+k t-n\right)
$$

donde $\frac{d n}{d t}$ es la tasa de producción de trampas de carga, $\Phi$ es la tasa de la dosis, $f$ es la fracción de trampas vacías convertidas en cargas atrapadas, $N_{0}$ es la concentración de trampas vacías ya presentes en el cristal antes de la irradiación, $k t$ es la cantidad de defectos producidos durante la radiación en un tiempo $t$ y $n$ es la concentración de cargas atrapadas en el tiempo $t$. La solución de esta ecuación considerando $n=0$ en $t=0$, está entonces dada por:

$$
n=\left(N_{0}-\frac{k}{\Phi f}\right)[1-\exp (-\Phi f t)]+k t
$$

la cual tiene dos componentes, una de saturación exponencial y otra lineal. La ecuación (2) puede ser modificada considerando que durante la radiación un cierto número de trampas vacías presentes antes de la radiación, $N_{0}$, puede ser modificado por la radiación en sí misma. El aumento y la disminución de la concentración de defectos puede ser observada como la suma de las componentes de saturación de incremento y disminución.

La probabilidad $p$ por unidad de tiempo de que un electrón atrapado escape de la trampa, está dada como:

$$
p=s \cdot \exp \left(-\frac{E}{k T}\right)
$$


donde $s$ es el factor de frecuencia $\left(\mathrm{s}^{-1}\right), E$ es la energía térmica de activación requerida para liberar un portador de carga atrapado, llamada también profundidad de la trampa $(\mathrm{eV}), k$ es la constante de Boltzmann y $T$ es la temperatura absoluta (K). El factor $s$ representa la frecuencia de vibración de la red y tiene valores generalmente que están en el orden de $10^{12}-10^{14} \mathrm{~s}^{-1}$. El tiempo de vida $\tau$, de los portadores de carga en el estado metaestable a la temperatura $T$ está dado por $\tau=p^{-1}$.

La suposición básica de que ningún electrón liberado de una trampa es reatrapado conduce al concepto de cinética de primer orden. Si $n$ es el número de electrones atrapados en el material y si la temperatura es mantenida constante, entonces $n$ decrece con el tiempo de acuerdo con la siguiente expresión:

$$
\frac{d n}{d t}=-p \cdot n
$$

Integrando la ecuación (4) y usando la ecuación (3), se tiene que:

$$
n=n_{0} \exp \left(-s t \cdot \exp \left(-\frac{E}{k T}\right)\right)
$$

donde $n_{0}$ es el número de electrones atrapados en $t_{0}=0$.

\subsection{Cinética de primer orden}

Consideremos una representación matemática para cada pico en una curva de brillo, con las siguientes suposiciones [17]:

- Irradiación del material a temperatura suficientemente baja para que ningún electrón sea liberado.

- Calentamiento a una tasa de temperatura constante.

La teoría de Randall-Wilkins asume un primer orden cinético y una única profundidad de trampa. Así, la intensidad termoluminiscente $I$, a cualquier temperatura $T$ es directamente proporcional a la tasa de liberación de cargas, y está dada como:

$$
I(T)=n_{0} s \exp \left(-\frac{E}{k T}\right) \exp \left(-\left(\frac{s}{\beta}\right) \int_{T_{0}}^{T} \exp \left(-\frac{E}{k T^{\prime}}\right) d T^{\prime}\right),
$$

donde $\beta$ es la tasa de calentamiento.

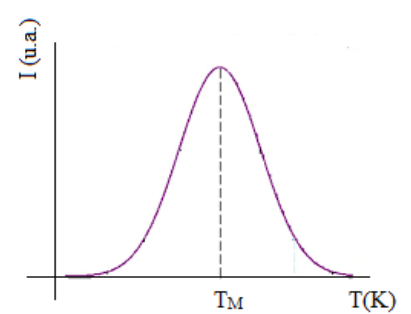

Fig. 1. Curva-solución de la ecuación (6). $T_{M}$ es independiente de la concentración inicial de electrones atrapados $n_{0}$. 
Esta expresión puede ser evaluada por integración numérica y su resultado es una curva en forma de campana puntiaguda, conocida como curva de brillo, con máximo de intensidad a una temperatura característica $T_{M}$, como muestra la Figura 1.

Una relación importante que se obtiene al ajustar la Figura 1 es $\frac{d I}{d T}=0$ para $T=T_{M}$.

De la ecuación (4) se obtiene que:

$$
\ln (I)=\ln \left(n_{0} s\right)-\frac{E}{k T}-\left(\frac{s}{\beta}\right) \int_{T_{0}}^{T} \exp \left(-\frac{E}{k T^{\prime}}\right) d T^{\prime},
$$

de donde derivando respecto de $T$ se llega a:

$$
\frac{d \ln (I)}{d T}=\frac{E}{k T^{2}}-\frac{s}{\beta} \exp \left(-\frac{E}{k T}\right),
$$

y considerando la condición $T=T_{M}$, se obtiene la expresión

$$
\frac{\beta E}{k T_{M}^{2}}=s \exp \left(-\frac{E}{k T_{M}}\right) \text {. }
$$

De las ecuaciones anteriores se obtienen algunas conclusiones importantes:

- Para una trampa dada $E$ y $s$ son valores constantes y $T_{M}$ se mueve hacia una mayor temperatura cuando la tasa de calentamiento aumenta.

- $T_{M}$ es independiente de $n_{0}$.

- La suma $S$ de la integral de la curva de brillo está dada por:

$$
S=\int_{0}^{\infty} I d t=-c \int_{n_{0}}^{0} d n=c n_{0}
$$

esto es, $S$ es proporcional al número inicial de cargas atrapadas, el cual a su vez es proporcional a la dosis de radiación.

Otras generalizaciones, como cinéticas de segundo orden y de orden general pueden ser consideradas cuando los fenómenos de reatrapamiento de portadores de carga llegan a ser dominantes [18].

\section{Procedimiento experimental}

Se analizaron ocho muestras, de las cuales siete fueron sintetizadas a base $\mathrm{Y}_{2} \mathrm{O}_{3}$ y la restante fue un dosímetro comercial TLD-100 (LiF:Mg,Ti); este último fue utilizado a manera de comparación. Para la caracterización, las muestras se sometieron inicialmente a un tratamiento térmico de borrado, con la finalidad de eliminar señales espurias en el material, el cual se desarrolló en tres etapas: Primero las muestras se mantuvieron durante 1 hora a $400 \pm 1^{\circ} \mathrm{C}$ en una mufla Terlab MA12D, luego se dejaron por 20 minutos a temperatura ambiente en un contenedor metálico, y posteriormente se empleó un horno Binder ED23, donde se mantuvieron a $100 \pm 1^{\circ} \mathrm{C}$ por 2 horas.

Posteriormente las muestras fueron irradias con una máquina de rayos $\mathrm{X}$ marca Siemens. Se emplearon valores de exposición en el rango de 17.7 a $40.0 \mathrm{R}$. La exposición se midió empleando una cámara de ionización Unfors ThinX RAD (Billdal, 
Suecia). Las muestras fueron irradiadas cinco veces, cada vez con niveles de exposición diferentes. Véase la Tabla 1.

Tabla 1. Parámetros de irradiación de la máquina de rayos $\mathrm{X}$.

\begin{tabular}{|c|c|c|}
\hline Kilovoltaje $(\mathrm{kV})$ & Carga de Trabajo (mAs) & Exposición $(\mathrm{R})$ \\
\hline 53.5 & 5.6 & 17.7 \\
\hline 70.0 & 12.5 & 23.1 \\
\hline 81.0 & 20.0 & 26.8 \\
\hline 109.0 & 32.0 & 36.0 \\
\hline 121.0 & 80.0 & 40.0 \\
\hline
\end{tabular}

Finalmente se llevó a cabo el proceso de lectura, empleando un lector Harshaw TLD 3500. Los datos fueron procesados mediante el software WinREMS (Windows Radiation Evaluation and Management System). Se obtuvieron las curvas de brillo para cada muestra.

\section{Determinación de los parámetros de la curva de brillo por el método de los tres puntos}

A partir de la curva TL experimental del material y tomando en cuenta la forma inicial del pico TL, fue posible determinar los parámetros cinéticos asociados a las curvas de brillo, esto es: Orden de la cinética $b$, energía de activación $E(\mathrm{eV})$ y factor de frecuencia $s\left(\mathrm{~s}^{-1}\right)[19,20]$.

En este trabajo se empleó el método de los tres puntos para obtener la deconvolución de la curva de brillo y los parámetros cinéticos de los picos.

Consideremos la intensidad máxima del pico, la cual puede obtenerse como:

$$
I_{M}=\frac{\left(A_{M}\right)^{b}}{N^{b-1}} s \exp \left(-\frac{E}{k T_{M}}\right)
$$

donde $A_{M}$ es el área bajo la mitad derecha del pico, entre $T_{M}$ y la temperatura final $T_{f}$. Definamos además las intensidades $\frac{I_{M}}{2}$ e $\frac{I_{M}}{4}$ de la forma:

$$
\begin{gathered}
\frac{I_{M}}{2}=\frac{\left(A_{2}\right)^{b}}{N^{b-1}} s \exp \left(-\frac{E}{k T_{2}}\right), \\
\frac{I_{M}}{4}=\frac{\left(A_{4}\right)^{b}}{N^{b-1}} s \exp \left(-\frac{E}{k T_{4}}\right),
\end{gathered}
$$

donde $A_{2}$ y $A_{4}$ son las porciones de áreas de la curva definidas entre las temperaturas $T_{2}$ y $T_{4}$ y la temperatura final $T_{f}$ del pico, como se muestra en la Figura 2. Combinando las ecuaciones (11) y (12) se obtiene la expresión para la energía de activación $E$ : 
Determinación de los parámetros de la curva de brillo de nanocristales de óxidos de itrio...

$$
E=\left[\ln 2-b \ln \left(\frac{A_{M}}{A_{2}}\right)\right]\left[\begin{array}{c}
k T_{M} T_{2} \\
T_{M}-T_{2}
\end{array}\right]
$$

Similarmente, de las ecuaciones (11) y (13) se obtiene

$$
E=\left[\ln 4-b\left(\frac{A_{M}}{A_{4}}\right)\right]\left[\frac{k T_{M} T_{4}}{T_{M}-T_{4}}\right] .
$$

Despejando $b$ de las ecuaciones (14) y (15) se obtiene que

$$
b=\frac{T_{2}\left(T_{M}-T_{4}\right) \ln 2-T_{4}\left(T_{M}-T_{2}\right) \ln 4}{T_{2}\left(T_{M}-T_{4}\right) \ln \left(\frac{A_{M}}{A_{2}}\right)-T_{4}\left(T_{M}-T_{2}\right) \ln \left(\frac{A_{M}}{A_{4}}\right)} .
$$

Por otro lado, el factor de frecuencia se obtiene en forma directa de la ecuación (9), esto es:

$$
s=\frac{\beta E}{k T_{M}^{2}} \exp \left(\frac{E}{k T_{M}}\right)
$$

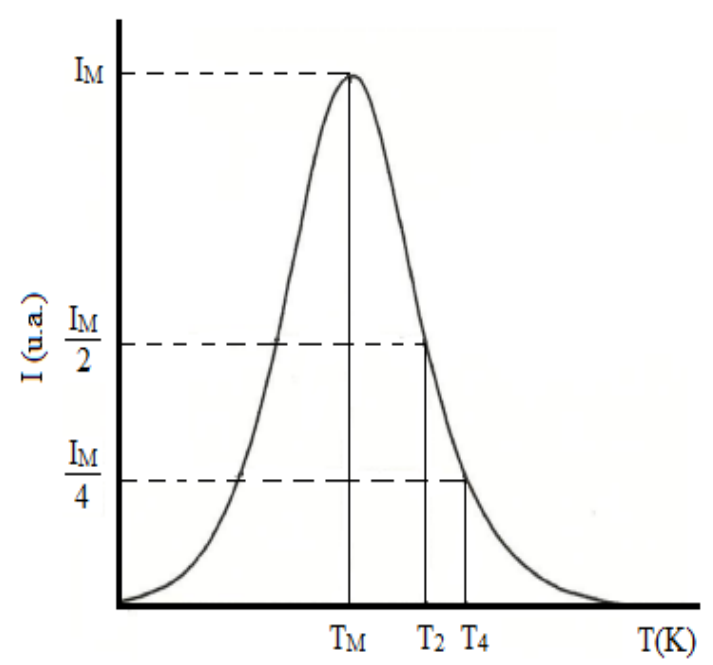

Fig. 2. Curva de brillo ideal mostrando los tres puntos $\left(T_{M}, I_{M}\right),\left(T_{2}, I_{M / 2}\right)$ y $\left(T_{4}, I_{M / 4}\right)$.

\subsection{Ejemplo de deconvolución de una curva de brillo}

Aplicamos el procedimiento descrito a la curva de brillo que se muestra en la Figura 3 , correspondiente a una muestra de $\mathrm{Y}_{2} \mathrm{O}_{3}:$ Ce $0.5 \%$, irradiada con una exposición de $36 \mathrm{R}$. 
Francisco del Rosario Sánchez, Modesto Sosa

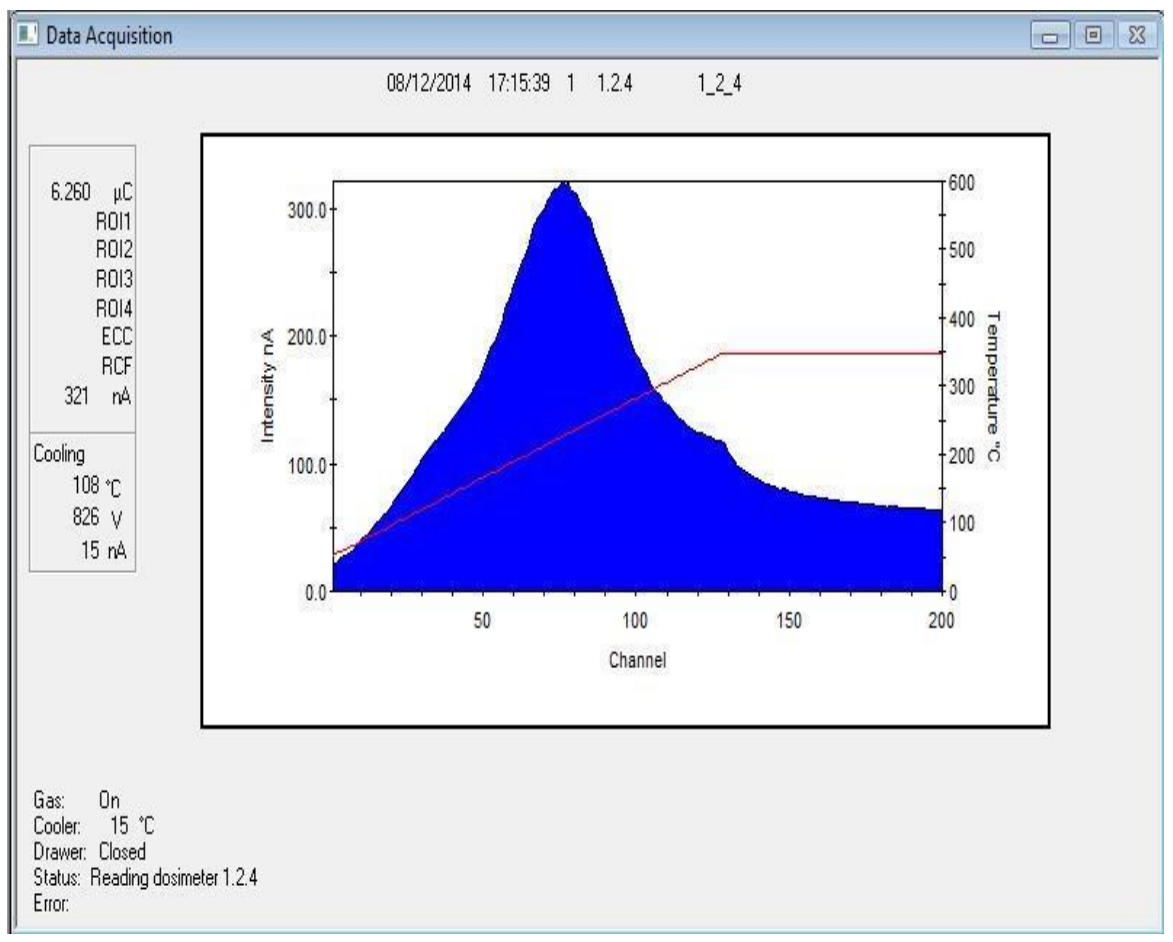

Fig. 3. Curva de brillo de una muestra de $\mathrm{Y}_{2} \mathrm{O}_{3}$ : Ce 0.5\%, generada por el lector Harshaw.

Aplicando el software WebPlotDigitizer v3.8 a la Figura 3, obtenemos la tabla de valores para su reconstrucción. La Figura 4 muestra la curva reconstruida.

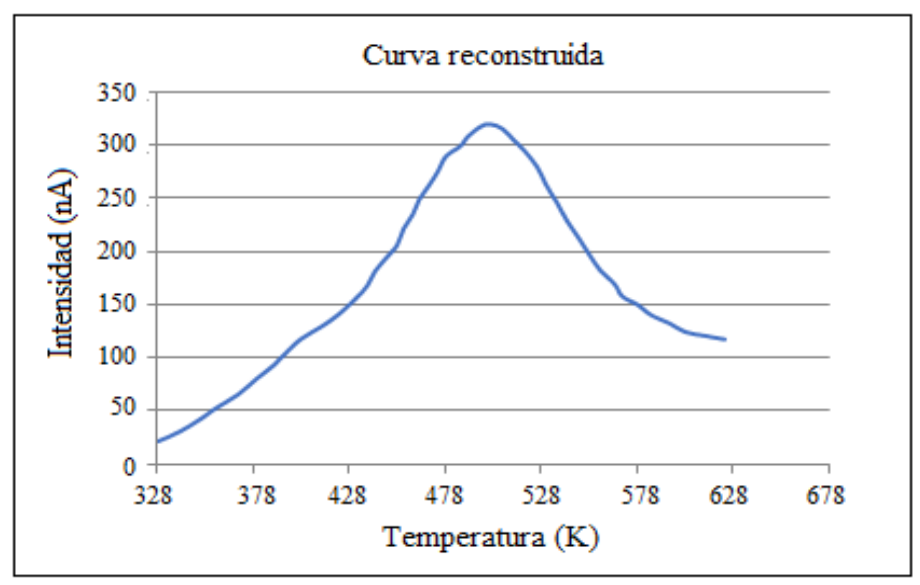

Fig. 4. Curva de brillo reconstruida a partir de los datos obtenidos. 
Nótese que aparentemente esta curva tiene un solo pico; no obstante, el modelo proporciona información de varios picos constituyentes, además del ruido. La Figura 5a muestra el pico principal (pico 1) de la curva de brillo extraído por el modelo.

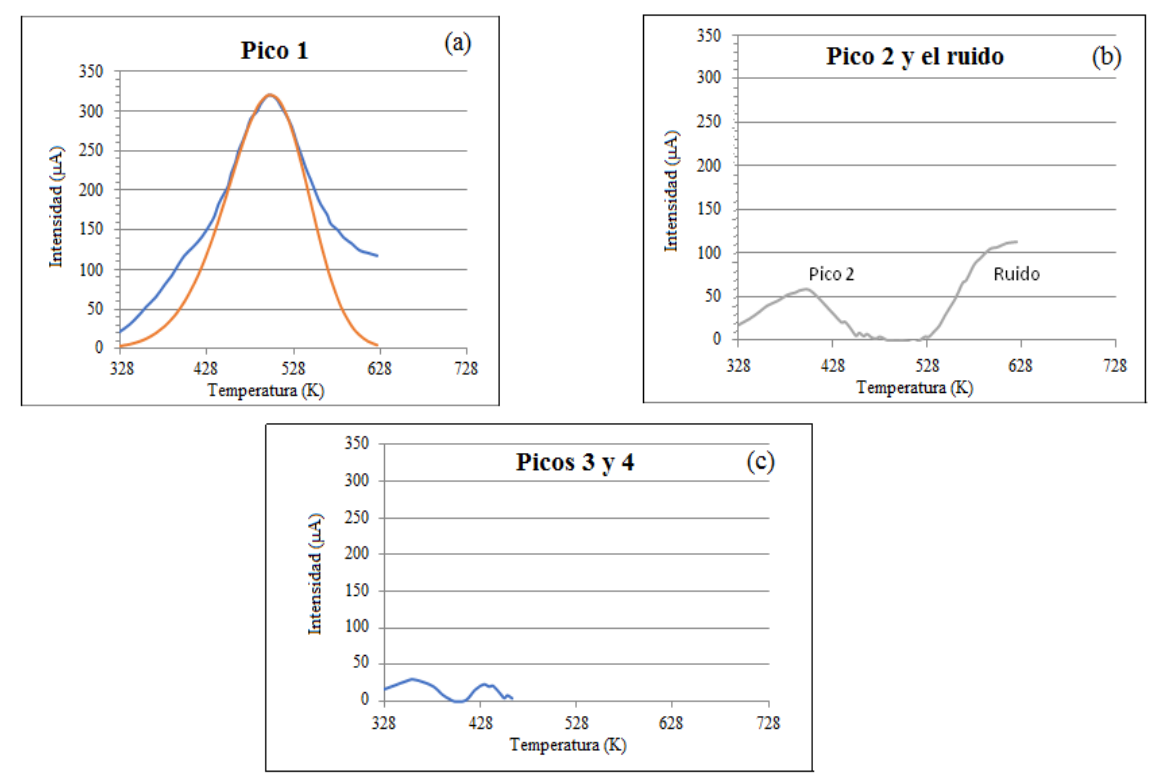

Fig. 5. Picos de la curva de brillo reconstruidos a partir de los datos obtenidos.

$\mathrm{Al}$ extraer el primer pico de la curva queda la señal residual mostrada en la Figura 5 b, en la cual se observa un pico (pico 2) de más baja temperatura y ruido a más alta temperatura. Si se aplica el modelo nuevamente al pico 2 y se extrae, se obtiene la señal mostrada en la Figura 5c, donde se observan dos picos de más baja intensidad (picos 3 y 4). A partir de las figuras anteriores se extraen los parámetros de la curva de brillo, los cuales se muestran en la Tabla 2.

Tabla 2. Parámetros cinéticos de la curva de brillo mostrada en la Figura 3.

\begin{tabular}{|l|l|l|}
\hline Parámetros cinéticos & Pico 1 & Pico 2 \\
\hline$T_{M}(\mathrm{~K})$ & 501.14 & 402.57 \\
\hline$I_{M}(\mu \mathrm{A})$ & 320.35 & 57.65 \\
\hline$T_{0}(\mathrm{~K})$ & 327.82 & 327.82 \\
\hline$\beta\left(\mathrm{K} \cdot \mathrm{s}^{-1}\right)$ & 10.00 & 10.00 \\
\hline $\mathrm{b}$ & 1.11 & 1.00 \\
\hline$E(\mathrm{eV})$ & 0.45 & 0.71 \\
\hline
\end{tabular}




\section{Discusión y conclusiones}

Se ha estudiado la respuesta termoluminiscente de diversos materiales al ser irradiados con rayos $\mathrm{X}$, y se han determinado los parámetros que caracterizan el orden de la cinética del material y la profundidad de trampas. Se ha empleado el método de los tres puntos para analizar la curva de brillo, la cual fue previamente reconstruida a partir de un proceso de deconvolución desarrollado en este trabajo usando el software WebPlotDigitizer v3.8. El análisis realizado muestra que en los materiales estudiados los procesos de recombinación predominan sobre los de reatrapamiento, tal como se deduce del parámetro cinético $b$ (ver tabla 2), que corresponde a una cinética cercana a primer orden. Por otra parte, el cálculo de la energía de activación arroja valores cercanos a $1 \mathrm{eV}$, lo cual está en concordancia con los reportados en la literatura para la mayoría de los materiales termoluminiscentes.

Agradecimientos. Este trabajo fue parcialmente financiado por el Consejo Nacional de Ciencia y Tecnología (CONACyT), proyecto 257599- CB2015 y por la DAIP-UG, convocatoria institucional 2018.

\section{Referencias}

1. Daniels, F., Boyd, C.A., Saunders, D.F.: Thermoluminescence as a research tool. Science 117, 343-349 (1953)

2. Kron, T.: Applications of thermoluminescence dosimetry in medicine. Radiat. Prot. Dosimetry 85, 333-340 (1999)

3. Bartolotta, A., Brai, M., Caputo, V., Di Liberto, R., Di Mariano, D., Ferrara, G., Puccio, P., Santamaria, A.S.: The response behaviour of LiF:Mg,Cu,P thermoluminescence dosimeters to high-energy electron beams used in radiotherapy. Phys. Med. Biol. 40, 211-220 (1995)

4. Belmonte, A.D., Baraja, J.M.D., Manso, M.L., del Rio, J.R.S.: Exit dose as a method to verify external radiotherapy treatments in vivo by TLD'S. Physica Medica 17, 7-9 (2001)

5. Essers, M., Minjheer, B.J.: In vivo dosimetry during external photon beam radiotherapy. Int. J. Radiat. Oncology Biol. Phys. 43, 245-259 (1999)

6. Azorín, J., Furetta, C., Scacco, A.: Preparation and properties of thermoluminescent materials. Physica Status Solidi 138, 9-46 (1993)

7. McKeever, S.W.S., Rhodes, J.F., Mathur, V.K., Chen, R., Brown, M.D., Bull, R.K.: Numerical solutions to the rate equations governing the simultaneous release of electrons and holes during thermoluminescence and isothermal decay. Phys. Rev. B32, 38353843 (1985)

8. McKeever, S.W.S., Chen, R.: Luminescence models. Radiat. Meas. 27, 625-661 (1997)

9. Pagonis, V., Mian, S., Kitis, G.: Fit of first order thermoluminescence glow peaks using the Weibull distribution function. Radiat. Prot. Dosimetry 93, 11-17 (2001)

10. Pagonis, V., Kitis, G.: Fit of second order thermoluminescence glow peaks using the Logistic distribution function. Radiat. Prot. Dosimetry 95, 225-229 (2001)

11. Pagonis, V., Kitis, G.: On the possibility of using commercial software packages for thermoluminescence glow curve deconvolution analysis. Radiat. Prot. Dosimetry 101, 9398 (2002)

12. Kitis, G., Gomez-Ros, J.M., Tuyn, J.W.N.: Thermoluminescence glow-curve deconvolution functions for first, second and general orders of kinetics. J. Phys. D31, 26362641 (1998) 
13. Osorio, E., Gutierrez, O.D., Paucar, C.G., Hadad, C.Z.: Thermoluminescence glow curves analysis of pure and $\mathrm{CeO}_{2}$-doped $\mathrm{Li}_{2} \mathrm{O}-\mathrm{Al}_{2} \mathrm{O}_{3}-\mathrm{SiO}_{2}$ glass ceramics. J. Luminescence 129, 657-660 (2009)

14. Phan, T.L., Phan, M.H., Vu, N., Anh, T.K., Yu, S.C.: Luminescent properties of Eu-doped $\mathrm{Y}_{2} \mathrm{O}_{3}$ nanophosphors. Physica Status Solidi 201, 2170-2174 (2004)

15. Jacobsohn, L.G., Blair, M.W., Tomga, S.C., Brown, L.O., Bennett, B.L., Muenchausen, R.E.: Y2O3:Bi nanophosphor: Solution combustion synthesis, structure, and luminescence. J. Appl. Phys. 104, 124303 (2008)

16. Furetta, C., Weng, P.S.: Operational Thermolumiscence Dosimetry. World Scientific, Singapore (1988)

17. Randall, J.T., Wilkins, M.H.F.: Phosphorescence and electron traps I. The study of trap distributions. In: Proc. R. Soc. No. 184 (noviembre, 1945a), pp. 366-389 (1945)

18. Garlick, G.F.J., Gibson, A.F.: Proc. Phys. Soc. 60, 574 (1948)

19. Rasheedy, M.S.: A new evaluation technique for analyzing the thermoluminescence glow curve and calculating the trap parameters. Thermochimica Acta 429, 143-147 (2005)

20. Rasheedy, M.S., El-Sherif, M.A., Hefnia, M.A.: Applications of the three points analysis method for obtaining the trap parameters and the separation of thermoluminescence glow curve into its components. Radiation Effects \& Defects in Solids 161, 579-590 (2006) 